\title{
The Dark Matter filament between Abell 222/223*
}

\author{
Jörg P. Dietrich ${ }^{1}$, Norbert Werner ${ }^{2}$, Douglas Clowe ${ }^{3}$, Alexis \\ Finoguenov $^{4}$, Tom Kitching ${ }^{5}$, Lance Miller ${ }^{6}$ and Aurora Simionescu ${ }^{2}$ \\ ${ }^{1}$ Universitäts-Sternwarte München, Ludwig-Maximilians-Universität München, Scheinerstr. 1, \\ 81679 München, Germany \\ email: dietrich@usm.lmu.de \\ ${ }^{2}$ Kavli Institute for Particle Astrophysics and Cosmology, Stanford University, 382 Via Pueblo \\ Mall, Stanford, CA 94305-4060, USA \\ ${ }^{3}$ Dept. of Physics \& Astronomy, Ohio University, Clippinger Lab 251B, Athens, OH 45701, \\ USA \\ ${ }^{4}$ Max-Planck-Institut fur extraterrestrische Physik, Giessenbachstraße, 85748 Garching b. \\ München, Germany \\ ${ }^{5}$ Institute for Astronomy, The University of Edinburgh, Royal Observatory, Blackford Hill, \\ Edinburgh EH9 3HJ, U.K. \\ ${ }^{6}$ Department of Physics, University of Oxford, The Denys Wilkinson Building, Keble Road, \\ Oxford OX1 3RH, U.K.
}

\begin{abstract}
Weak lensing detections and measurements of filaments have been elusive for a long time. The reason is that the low density contrast of filaments generally pushes the weak lensing signal to unobservably low scales. To nevertheless map the dark matter in filaments exquisite data and unusual systems are necessary. SuprimeCam observations of the supercluster system Abell 222/223 provided the required combination of excellent seeing images and a fortuitous alignment of the filament with the line-of-sight. This boosted the lensing signal to a detectable level and led to the first weak lensing mass measurement of a large-scale structure filament. The filament connecting Abell 222 and Abell 223 is now the only one traced by the galaxy distribution, dark matter, and X-ray emission from the hottest phase of the warm-hot intergalactic medium. The combination of these data allows us to put the first constraints on the hot gas fraction in filaments.
\end{abstract}

Keywords. gravitational lensing: weak, galaxies: clusters: individual (Abell 222, Abell 223), large-scale structure of universe

It is a firm prediction of the concordance Cold Dark Matter (CDM) cosmological model that galaxy clusters live at the intersection of large-scale structure filaments (Bond et al. 1996). The thread-like structure of this "cosmic web" has been traced by galaxy redshift surveys for decades (e.g. Joeveer et al. 1978; Geller \& Huchra 1989). More recently the Warm-Hot Intergalactic Medium (WHIM) residing in low redshift filaments has been observed in emission (Werner et al. 2008) and absorption (Buote et al. 2009; Fang et al. 2010). However, a reliable direct detection of the underlying Dark Matter skeleton, which should contain more than half of all matter (Aragón-Calvo et al. 2010), remained elusive for much longer, as earlier candidates for such detections (Kaiser et al. 1998; Gray et al. 2002; Dietrich et al. 2005) were either falsified (Gavazzi et al. 2004; Heymans et al. 2008) or suffered from low signal-to-noise ratios (Kaiser et al. 1998; Dietrich et al. 2005) and

* The copyright line contained an error. This has now been corrected in the online version, with an additional correction notice published. 
unphysical misalignements of dark and luminous matter (Gray et al. 2002; Dietrich et al. 2005).

Abell 222 and Abell 223, the latter a double galaxy cluster in itself, form a supercluster system of three galaxy clusters at a redshift of $z \sim 0.21$ (Dietrich et al. 2002), separated on the sky by $\sim 14^{\prime}$. Gravitational lensing distorts the images of faint background galaxies as their light passes massive foreground structures. The foreground mass and its distribution can be deduced from measuring the shear field imprinted on the shapes of the background galaxies. The mass reconstruction in Figure 1 shows a mass bridge connecting A 222 and the southern component of A 223 (A 223-S) at the $4.1 \sigma$ significance level. This mass reconstruction does not assume any model or physical prior on the mass distribution.

To show that the mass bridge extending between A 222 and A 223 is not caused by the overlap of the cluster halos but in fact due to additional mass, we also fit parametric models to the three clusters plus a filament component. The clusters were modelled as elliptical Navarro-Frenk-White (NFW) profiles (Navarro et al. 1997) with a fixed massconcentration relation (Dolag et al. 2004). We used a simple model for the filament, with a flat ridge line connecting the clusters, exponential cut-offs at the filament end points in the clusters, and a King profile (King 1966) describing the radial density distribution, as suggested by previous studies (Colberg et al. 2005; Mead et al. 2010). We showed in the original publication of this work (Dietrich et al. 2012) that the exact ellipticity has little impact on the significance of the filament.

The best fit parameters of this model were determined with a Monte-Carlo Markov Chain (MCMC) and are shown in Fig. 2. The likelihood-ratio test prefers models with a filament component with $96.0 \%$ confidence over a fit with three NFW halos only. A small degeneracy exists in the model between the strength of the filament and the virial radii of A 222 and A 223-S. The fitting procedure tries to keep the total amount of mass in the supercluster system constant at the level indicated by the observed reduced shear. Thus, it is not necessarily the case that sample points with a positive filament contribution indeed have more mass in the filament area than a 3 clusters only model has. The reason is that the additional filament mass might be compensated for with lower cluster masses. We find that the integrated surface mass density along the filament ridge line exceeds that of the clusters only model in $98.5 \%$ of all sample points. This indicates that the data strongly prefers models with additional mass between A 222 and A 223-S and that this preference is stronger than the confidence level derived from the likelihood-ratio test. The difference is probably due to the oversimplified model, which is not a good representation of the true filament shape.

The virial masses inferred from the MCMC are lower than those reported earlier for this system (Dietrich et al. 2005), which were obtained from fitting a circular two-component NFW model to A 222 and A 223. Compared to this approach, our more complex model removes mass from the individual supercluster constituents and redistributes it to the filament component. Reproducing the two-component fit with free concentration parameters, which was used in the previous study, we find $M_{200}(\mathrm{~A} 222)=\left(2.7_{-0.7}^{+0.8}\right) \times 10^{14} M_{\odot}$, which is in good agreement, and $M_{200}(\mathrm{~A} 223)=\left(3.4_{-1.0}^{+1.3}\right) \times 10^{14} M_{\odot}$, which overlaps the $1 \sigma$ error bars of the earlier study. Here and in the following, all error bars are single standard deviations.

The detection of a filament with a dimensionless surface mass density of $\kappa \sim 0.03$ is unexpected. Simulations generally predict the surface mass density of filaments to be much lower (Dietrich et al. 2005) and not to be detectable individually (Mead et al. 2010). These predictions, however, are based on the assumption that the longer axis of the filament is aligned with the plane of the sky and that we look through the filament 


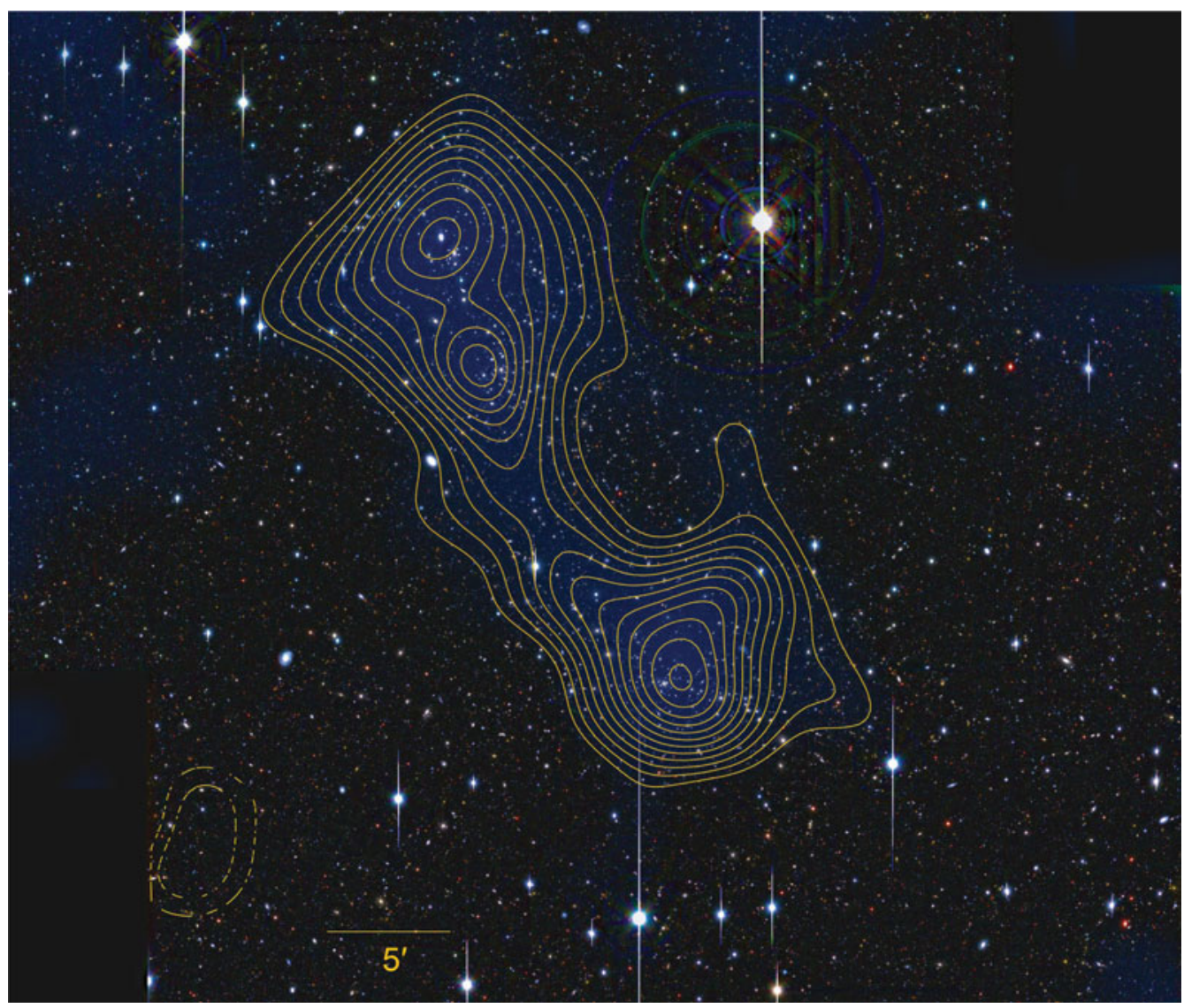

Figure 1. Mass reconstruction of A 222/223. The background image is a three colour-composite SuprimeCam image based on observations with the $8 \mathrm{~m}$ Subaru telescope during the nights of Oct. 15 (A 222) and 20 (A 223), 2001 in V-, $R_{c^{-}}$and i'-bands. We obtained the data from the SMOKA science archive (http://smoka.nao.ac.jp/). The FWHM of the stellar point-spread function varies between $0 . .57$ and $00^{\prime \prime} 70$ in our final co-added images. Overlayed are the reconstructed surface mass density (blue) above $\kappa=0.0077$, corresponding to $\Sigma=2.36 M_{\odot} \mathrm{Mpc}^{-2}$, and significance contours above the mean of the field edge, rising in steps of $0.5 \sigma$ and starting from 2.5 $\sigma$. Dashed contours mark underdense regions at the same significance levels. Supplementary Figure 1 shows the corresponding B-mode map. The reconstruction is based on 40,341 galaxies whose colours are not consistent with early type galaxies at the cluster redshift. The shear field was smoothed with a $2^{\prime}$ Gaussian. The significance was assessed from the variance of 800 mass maps created from catalogues with randomised background galaxy orientation. We measured the shapes of these galaxies primarily in the $R_{c}$-band, supplementing the galaxy shape catalogue with measurements from the other two bands for galaxies for which no shapes could be measured in the $\mathrm{R}_{\mathrm{c}}$-band, to estimate the gravitational shear (Miller et al. 2007; Kitching et al. 2008). A 222 is detected at $\sim 8.0 \sigma$ in the south, A 223 is the double-peaked structure in the north seen at $\sim 7 \sigma$.

along its minor axis. If the filament were inclined with respect to the line-of-sight and we were to look almost along its major axis, the projected mass could reach the observed level. A timing argument (Kahn \& Woltjer 1959; Sandage 1986) can be made to show that the latter scenario is more plausible in the A $222 / 3$ system. In this argument we treat A 223 as a single cluster and neglect the filament component, such that we have to deal only with two bodies, A 222 and A 223. The redshifts of A 222 and A 223 differ by $\Delta z=0.005$, corresponding to a line-of-sight separation of $18 \mathrm{Mpc}$ if the redshift difference 


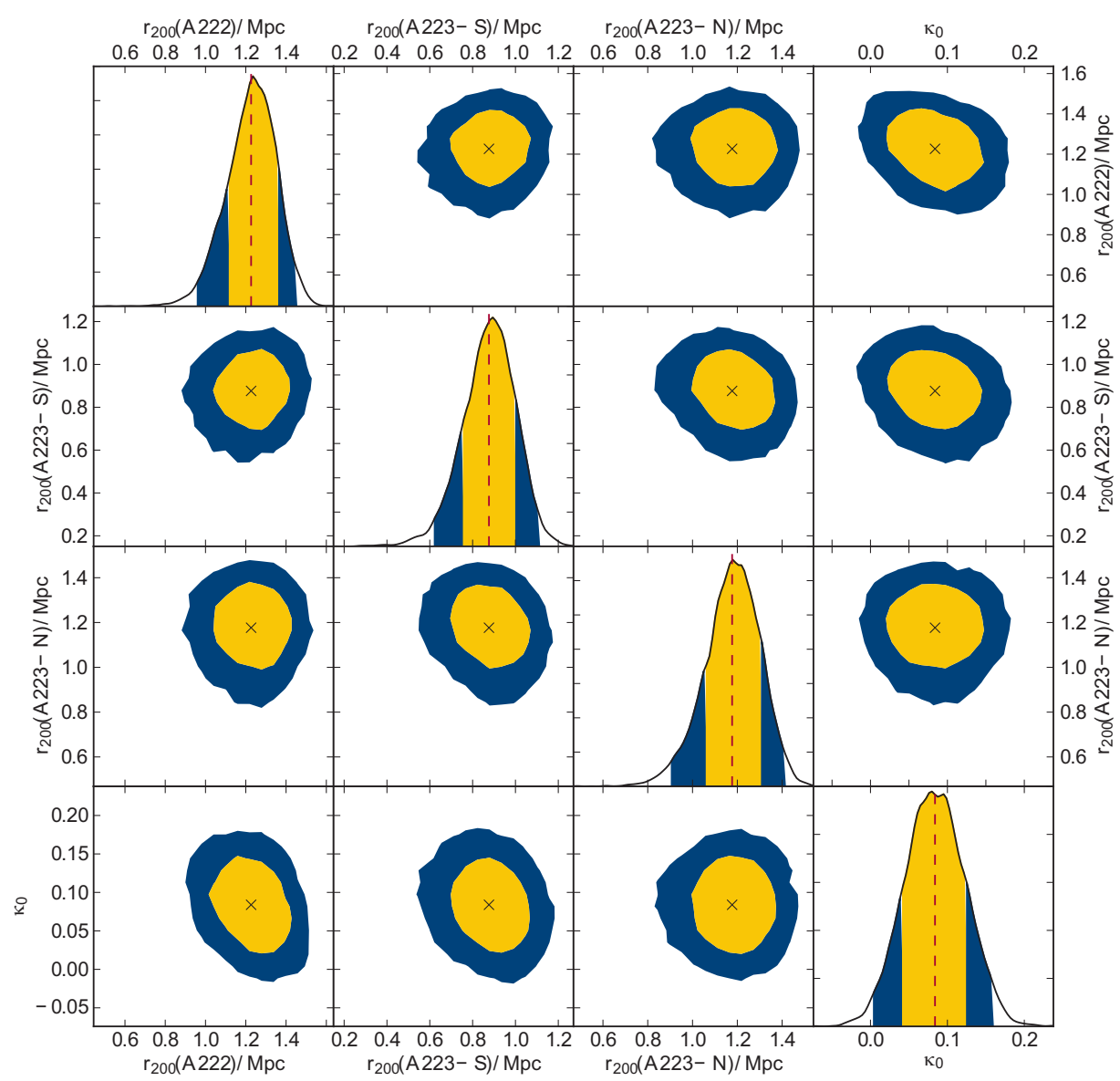

Figure 2. Posterior probability distributions for cluster virial radii and filament strength. Shown are the $68 \%$ and $95 \%$ confidence intervals on the cluster virial radii $r_{200}(\cdot)$ and the filament strength $\kappa_{0}$. The confidence intervals are derived from 30,000 MCMC sample points. The filament model is described by $\kappa(\theta, r)=\kappa_{0}\left\{1+\exp \left[\left(|\theta|-\theta_{1}\right) / \sigma\right]+\left(r / r_{\mathrm{c}}\right)^{2}\right\}^{-1}$, where the coordinate $\theta$ runs along the filament ridge line and $r$ is orthogonal to it. This model predicts the surface mass density at discrete grid points from which we computed our observable, the reduced shear, via a convolution in Fourier space. The data cannot constrain the steepness of the exponential cut-off at the filament endpoints $\sigma$ and the radial core scale $r_{\mathrm{c}}$. These were fixed at their approximate best-fit values of $\sigma=0.45 \mathrm{Mpc}$ and $r_{\mathrm{c}}=0.54 \mathrm{Mpc}$. The data also cannot constrain the cluster ellipticity and orientation. These were held fixed at the values measured from the isodensity contours of early-type galaxies (Dietrich et al. 2002). The ratios of minor/major axes and the position angles of the ellipses are $(0.63,0.69,0.70)$ and $\left(65^{\circ}, 34^{\circ}, 3^{\circ}\right)$ for A 222, A 223-S, and A 223-N, respectively. We further explore the impact of cluster ellipticity on the filament detection in the supplementary information.

is entirely due to Hubble flow. Let us assume for a moment that the difference is caused only by peculiar velocities. Then at $z=\infty$, the clusters were at the same location in the Hubble flow. We let them move away from each other with some velocity and inclination angle with respect to the line-of-sight and later turn around and approach each other. The parameter space of total system mass and inclination angle that reproduces the observed configuration at $z=0.21$ is completely degenerate. Nevertheless, in order to explain the observed configuration purely with peculiar velocity, this model requires a minimum mass of $(2.61 \pm 0.05) \times 10^{15} M_{\odot}$ with an inclination angle of 46 degrees, where 


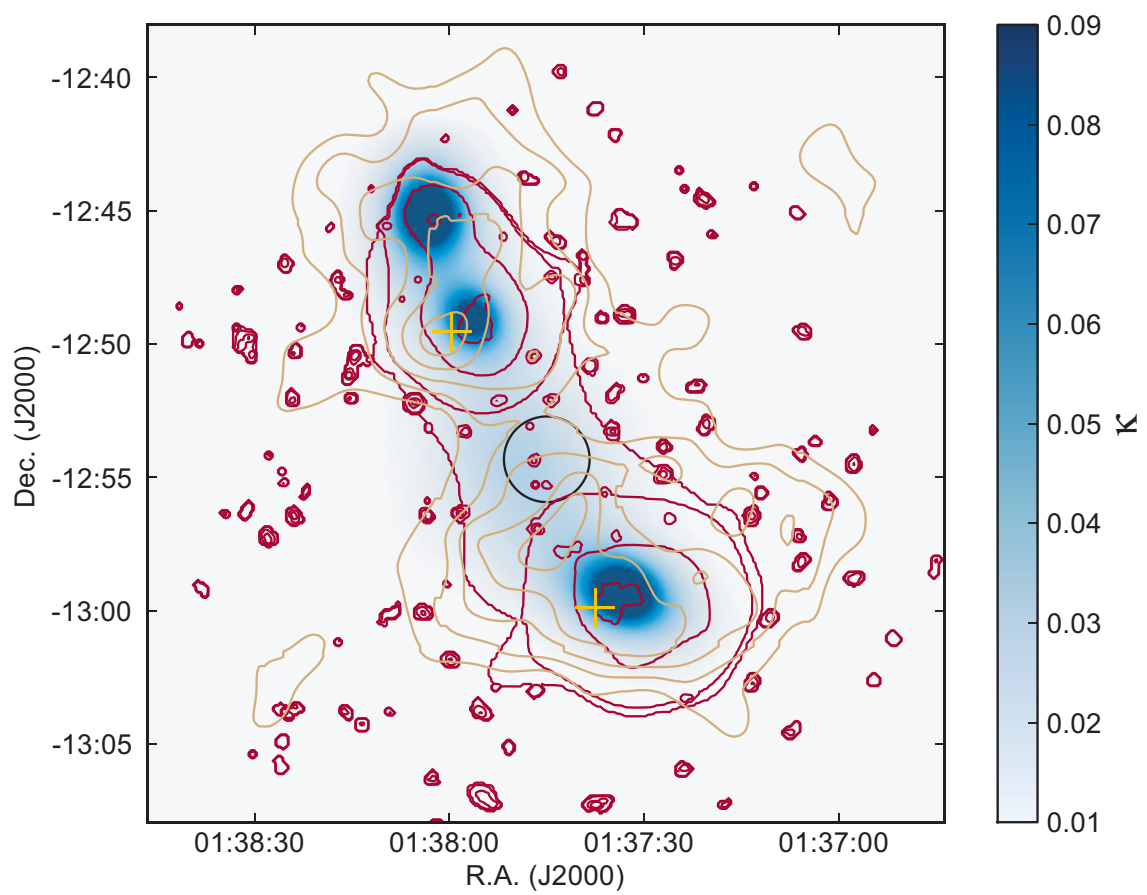

Figure 3. Surface mass density of the best fit parametric model. The surface mass density distribution of the best parameters in Fig. 2 was smoothed with a $2^{\prime}$ Gaussian to have the same physical resolution as the mass reconstruction in Fig. 1. The yellow crosses mark the end points of the filament model. These were determined from the visual impression of the filament axis in Fig. 1. The MCMC is not able to constrain their location. In the model, the filament ridge line is not aligned with the axis connecting the centers of A 222 and A 223-S. This is a fairly common occurrence $(\sim 9 \%)$ for straight filament but may also indicate some curvature, which occurs in $\sim 53 \%$ of all intercluster filaments (Colberg et al. 2005) and is not included in our simple model. Overlayed are X-ray contours from XMM-Newton observations (Werner et al. 2008) (red) and significance contours of the colour-selected early-type galaxy density (Dietrich et al. 2005) (beige), showing the alignment of all three filament constituents. The black circle marks the region inside which the gas mass and the filament mass were estimated.

the error on the mass is caused solely by the uncertainty of the Hubble constant. Since this is more than 10 standard deviations above our mass estimate for the sum of both clusters, we infer that at least part of the observed redshift difference is due to Hubble flow, and that we are looking along the filament's major axis.

The combination of our weak-lensing detection with the observed X-ray emission of $0.91 \pm 0.25 \mathrm{keV}$ WHIM plasma (Werner et al. 2008) lets us constrain the hot gas fraction in the filament. Assuming that the distribution of the hot plasma is uniform and adopting a metallicity of $Z=0.2$ Solar, the mass of the X-ray emitting gas inside a cylindrical region with radius $330 \mathrm{kpc}$ centred on $(01: 37: 45.00,12: 54: 19.6)$ with a length along our line-ofsight of $l=18 \mathrm{Mpc}$, as suggested by our timing argument, is $M_{\text {gas }}=5.8 \times 10^{12} M_{\odot}$. The assumption of uniform density is certainly a strong simplification. Because the $\mathrm{X}$-ray emissivity depends on the average of the squared gas density, a non-uniform density distribution can lead to strong changes in the X-ray luminosity. Thus, if the filament consists of denser clumps embedded into lower density gas (as has been observed in the outskirts of the Perseus Cluster (Simionescu et al. 2011)), or even if there is a smooth non-negligible density gradient within the region used for spectral extraction, then our 
best fit mean density will be overestimated. The quoted gas mass should therefore be considered as an upper limit, and the true mass may be lower by up to a factor of 2-3.

We estimated the total mass of the filament from the reconstructed surface massdensity map and the model fits within the same region where we measured the gas mass. In the reconstructed $\kappa$-map, the mass inside the extraction circle is $M_{\text {fil }}=(6.5 \pm 0.1) \times$ $10^{13} M_{\odot}$, where the error is small due to the highly correlated noise of the smoothed shear field inside the extraction aperture. For the parametric model fit, the inferred mass is higher but consistent within one standard deviation, $M_{\mathrm{fil}}=(9.8 \pm 4.4) \times 10^{13} M_{\odot}$. The corresponding upper limits on the hot gas fractions vary between $f_{\mathrm{X}}=0.06-0.09$, a value that is lower than the gas fraction in galaxy clusters (Allen et al. 2008). This is consistent with the expectation that a significant fraction of the WHIM in filaments is too cold to emit X-rays detectable by XMM-Newton (Davé et al. 2001).

The results reported in this proceeding were first published in Dietrich et al. (2012).

\section{References}

Allen, S. W., Rapetti, D. A., Schmidt, R. W., et al. 2008, Mon. Not. R. Astron. Soc., 383, 879 Aragón-Calvo, M. A., van de Weygaert, R., \& Jones, B. J. T. 2010, Mon. Not. R. Astron. Soc., 408,2163

Bond, J. R., Kofman, L., \& Pogosyan, D. 1996, Nature, 380, 603

Buote, D. A., Zappacosta, L., Fang, T., et al. 2009, Astrophys. J., 695, 1351

Colberg, J. M., Krughoff, K. S., \& Connolly, A. J. 2005, Mon. Not. R. Astron. Soc., 359, 272

Davé, R., Cen, R., Ostriker, J. P., et al. 2001, Astrophys. J., 552, 473

Dietrich, J. P., Clowe, D. I., \& Soucail, G. 2002, Astron. Astrophys., 394, 395

Dietrich, J. P., Schneider, P., Clowe, D., Romano-Díaz, E., \& Kerp, J. 2005, Astron. Astrophys., 440, 453

Dietrich, J. P., Werner, N., Clowe, D., et al. 2012, Nature, 487, 202

Dolag, K., Bartelmann, M., Perrotta, F., et al. 2004, Astron. Astrophys., 416, 853

Fang, T., Buote, D. A., Humphrey, P. J., et al. 2010, Astrophys. J., 714, 1715

Gavazzi, R., Mellier, Y., Fort, B., Cuillandre, J.-C., \& Dantel-Fort, M. 2004, Astron. Astrophys., 422,407

Geller, M. J. \& Huchra, J. P. 1989, Science, 246, 897

Gray, M. E., Taylor, A. N., Meisenheimer, K., et al. 2002, Astrophys. J., 568, 141

Heymans, C., Gray, M. E., Peng, C. Y., et al. 2008, Mon. Not. R. Astron. Soc., 385, 1431

Ilbert, O., Arnouts, S., McCracken, H. J., et al. 2006, Astron. Astrophys., 457, 841

Joeveer, M., Einasto, J., \& Tago, E. 1978, Mon. Not. R. Astron. Soc., 185, 357

Kahn, F. D. \& Woltjer, L. 1959, Astrophys. J., 130, 705

Kaiser, N., Wilson, G., Luppino, G., et al. 1998, astro-ph/9809268

King, I. R. 1966, Astron. J., 71, 64

Kitching, T. D., Miller, L., Heymans, C. E., van Waerbeke, L., \& Heavens, A. F. 2008, Mon. Not. R. Astron. Soc., 390, 149

Mead, J. M. G., King, L. J., \& McCarthy, I. G. 2010, Mon. Not. R. Astron. Soc., 401, 2257

Miller, L., Kitching, T. D., Heymans, C., Heavens, A. F., \& van Waerbeke, L. 2007, Mon. Not. R. Astron. Soc., 382, 315

Navarro, J. F., Frenk, C. S., \& White, S. D. M. 1997, Astrophys. J., 490, 493

Sandage, A. 1986, Astrophys. J., 307, 1

Simionescu, A., Allen, S. W., Mantz, A., et al. 2011, Science, 331, 1576

Werner, N., Finoguenov, A., Kaastra, J. S., et al. 2008, Astron. Astrophys., 482, L29 